\title{
On the Ionization of a Keplerian Binary System by Periodic Gravitational Radiation
}

\author{
C. Chicone* , B. Mashhoon ${ }^{\dagger}$ and D. G. Retzloff ${ }^{\ddagger}$
}

(June 23, 2021)

\begin{abstract}
The gravitational ionization of a Keplerian binary system via normally incident periodic gravitational radiation of definite helicity is discussed. The periodic orbits of the planar tidal equation are investigated on the basis of degenerate continuation theory. The relevance of the KolmogorovArnold-Moser theory to the question of gravitational ionization is elucidated, and it is conjectured that the process of ionization is closely related to the Arnold diffusion of the perturbed system.
\end{abstract}

04.20.Cv, 04.30.+x, 95.10.Ce

\section{INTRODUCTION}

In a recent papert, we considered the long-term nonlinear perturbations of Keplerian orbits by incident gravitational waves of wavelengths much larger than the size of the system. In particular, we studied the periodic orbits of the perturbed system using the methods developed in Ref. 2. The existence of periodic orbits indicates the possibility of balance in the exchange of energy between the binary and the external radiation field. Thus gravitational ionization does not occur for such orbits. The issue of gravitational ionization is interesting as it involves the transport of energy by gravitational radiation and is analogous to the corresponding phenomenon that is well known in the electromagnetic context.

The theoretical investigation of the interaction of a binary system with the gravitational radiation field reveals subtle phenomena that are further studied in this paper. In particular, the absorption of gravitational radiation energy by the binary is not unidirectional in general. That is, the orbital energy of a binary immersed in a gravitational radiation field does not in general increase monotonically with time. On the other hand, the emission of gravitational radiation by the binary is expected to be accompanied by the monotonic decrease of the orbital energy of the system. In absorption, however, the incident wave can deposit energy into the orbit during one time interval and remove energy from the orbit during another time interval. A periodic orbit would result - even when the emission of the radiation by the binary is ignored - if after a certain time the net flow of energy between the incident wave and the binary is zero.

Let us imagine, for the sake of simplicity, that gravitational radiation is incident on a Newtonian binary system consisting of a massive body of mass $M_{0}$ at the origin of inertial coordinates and a particle of test mass $m_{0}<<M_{0}$ that revolves around it in the $(x, y)$-plane. The dynamical equation in this case is of the general form 3 i

$$
\frac{d^{2} x^{i}}{d t^{2}}+\frac{k x^{i}}{r^{3}}+\epsilon \mathcal{K}_{i j}(t) x^{j}=0,
$$

where $k=G_{0}\left(M_{0}+m_{0}\right), \epsilon, 0<\epsilon<<1$, is the perturbation parameter and $\epsilon \mathcal{K}_{i j}$ is the tidal matrix associated with the incident gravitational waves. Here $\mathcal{K}$ is symmetric and traceless, and is related to the gravitational perturbation of the Minkowski space-time by

$$
\mathcal{K}_{i j}(t)=-\frac{1}{2} \frac{\partial^{2} \chi_{i j}}{\partial t^{2}}(t, \mathbf{0})
$$

where $g_{\mu \nu}=\eta_{\mu \nu}+\epsilon \chi_{\mu \nu}$. Here we employ the transverse-traceless gauge for gravitational radiation, i.e. $\chi_{0 \mu}=0, \chi_{i j}$ is traceless and $\partial_{j} \chi_{i j}=0$; moreover, $\chi_{i j}$ is a solution of the wave equation $\square \chi_{i j}=0$. Thus the gravitational radiation field can be expressed as a Fourier sum of monochromatic waves of frequency $\Omega_{\star}$ and wave vector $\mathbf{K}_{\star}, c\left|\mathbf{K}_{\star}\right|=\Omega_{\star}$,

$$
\chi_{i j}(t, \mathbf{x})=\operatorname{Re} \sum_{\mathbf{K}_{\star}} \widehat{\chi}_{i j}\left(\mathbf{K}_{\star}\right) \exp \left(i \mathbf{K}_{\star} \cdot \mathbf{x}-i \Omega_{\star} t\right),
$$

\footnotetext{
*Department of Mathematics, University of Missouri, Columbia, MO 65211. Supported by the NSF grant DMS-9303767.

${ }^{\dagger}$ Department of Physics and Astronomy, University of Missouri, Columbia, MO 65211.

${ }^{\ddagger}$ Department of Chemical Engineering, University of Missouri, Columbia, MO 65211.
} 
where $\widehat{\chi}_{i j}$ is symmetric, traceless and $\widehat{\chi}_{i j} K_{\star}^{j}=0$. The summation in (3) extends over all waves with $2 \pi c / \Omega_{\star}$ much larger than the average orbital radius. Equation (1) contains only the essential physics of the interaction of longwavelength gravitational radiation with a Newtonian binary system; in fact, relativistic (i.e. post-Keplerian) effects in the binary are totally neglected. In particular, the emission of gravitational waves is ignored. The motivation for our treatment as well as its limitations is presented in detail in our recent work

The incident wave exchanges energy and angular momentum with the binary orbit but not linear momentum in the quadrupole approximation under consideration here1. This is in exact analogy with the electromagnetic problem of the interaction of an electromagnetic wave with an atom in the dipole approximation.

A simple linear perturbation treatment of (11) has revealed the possibility of the existence of resonances at $\Omega_{\star}=m \omega$, $m=1,2,3, \cdots$, where $\omega$ is the Keplerian frequency of the unperturbed elliptifal orbit. Moreover, in this analysis

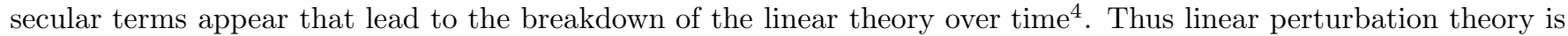
inappropriate for the investigation of periodic orbits of the perturbed system, since a periodic orbit is expected to persist forever beyond a certain point in time.

In the first treatment of the nonlinear case $₫$, we considered a single monochromatic plane wave of frequency $\Omega_{\star}$ that was normally incident on a Keplerian orbit of frequency $\omega$. We found that in the generic case, certain orbits satisfying the resonance condition $\Omega_{\star}=m \omega, m=1,2,3, \cdots$, could be continued to periodic orbits of the nonlinear system. The existence of periodic solutions of (11) demonstrates that ionization does not always occur; in fact, in a periodic orbit the energy exchange with the radiation field must be steady without any net flow. In addition, we found that for incident circularly polarized radiation of definite helicity the rotation of the inertial coordinates by frequency $\Omega_{\star} / 2$ rendered the dynamical equations autonomous. The invariance of this autonomous system under time translation implies the existence of an energy integral in the rotating frame. In this case, the Kolmogorov-Arnold-Moser (KAM) theory implies that for sufficiently small $\epsilon$ ionization can never occur in this system regardless of the magnitude of $\Omega_{\star} / \omega$. To understand intuitively how this could come about, it should be pointed out that a binary system can gain or lose energy as it interacts with an incident gravitational wave. The situation in absorption is in contrast to the emission of gravitational waves by a binary. In the latter situation, the binary is expected to lose energy monotonically; in fact, this is consistent with the observed rate of inward spiraling of the Hulse-Taylor binary pulsareld. It follows that the reciprocity between emission and absorption of gravitational waves does not hold in general. This notion of reciprocity is valid in some other situations, however. For instance, a Keplerian ellipse of frequency $\omega$ emits gravitational radiation of frequency $m \omega, m=1,2,3, \cdots$, which corresponds to the resonance condition for absorption.

Let us now consider a general periodic gravitational wave of period $2 \pi / \Omega_{\star}$ that is normally incident on the binary system. The existence of certain periodic solutions of the perturbed system may be expected on general grounds. It would therefore be more interesting to investigate the interaction of circularly polarized gravitational radiation with the binary system and to determine the stability of the resulting autonomous system under periodic perturbations. That is, a periodic wave may be expressed as a Fourier sum of components with frequencies $n \Omega_{\star}, n=1,2,3, \cdots$. For a single component of definite helicity, the transformation to the corresponding rotating frame would essentially remove the dependence of this perturbation upon time and for $\epsilon$ below a certain limit the orbit would remain forever bounded even though there is a steady flow of incident gravitational radiation energy in the inertial frame. However, the time-dependence of the other Fourier components would not disappear in the rotating frame, and we would like to study the influence of these components on the ionization of the system. Though we develop methods that are applicable to a general periodic perturbation, we restrict our attention to a tractable problem for the sake of simplicity.

In this paper, we consider a superposition of several harmonics in the perturbing function; clearly, the response of the system is not a superposition of the individual responses as a consequence of the intrinsic nonlinearity of the problem under consideration here. Specifically, we showed in Ref. 1 that for a normally incident circularly polarized monochromatic plane wave the motion is restricted to the $(x, y)$-plane and that a transformation to the uniformly rotating coordinate system in this plane with half the wave's frequency would result in an autonomous system for the equation of motion to which the KAM theory can be applied. It follows from the KAM theory that for sufficiently small $\epsilon$ the motion is confined and ionization does not occur. We wish to explore the sensitivity of this interesting result to the particular form of the incident wave. Therefore, we consider here a principal right circularly polarized wave of frequency $\Omega_{\star}=2 \Omega$ that is slightly modified by the presence of similar components of frequencies $\Omega$ and $3 \Omega$ as follows:

$$
\begin{aligned}
& \chi_{11}(t, \mathbf{0})=\cos 2 \Omega t+2 \delta\left[(\alpha-\beta) \cos \Omega t+\frac{1}{9}(\alpha+\beta) \cos 3 \Omega t\right], \\
& \chi_{12}(t, \mathbf{0})=\sin 2 \Omega t+2 \delta\left[(\alpha-\beta) \sin \Omega t+\frac{1}{9}(\alpha+\beta) \sin 3 \Omega t\right],
\end{aligned}
$$

$\chi_{13}=\chi_{23}=\chi_{33}=0$. The other components of $\chi$ follow from the fact that $\chi$ is a symmetric traceless matrix. Here $\delta$, $0<\delta<<1$, is a new perturbation parameter that determines the relative strength of the extra secondary components 
compared to the primary Fourier component of the normally incident radiation. Moreover, $\alpha$ and $\beta$ are constant amplitudes of the order of unity, and the other numerical coefficients have been introduced for the sake of simplicity.

The plan of this paper is as follows: In Section II, we present the basic equations for a Hamiltonian description of the perturbed orbit in terms of Delaunay variables. Sections III $\mathrm{V}$ are devoted to a development of degenerate continuation theory that is necessary for the identification of periodic orbits of the nonlinear problem via the methods and ideas that are originally due to Poincaré. The existence of periodic orbits, described in Section $\mathrm{V}$, demonstrates that a state of equilibrium can be established between the wave and the binary such that ionization does not occur; in fact, the net flow of energy vanishes in this case. To apply the KAM theory to our problem, it is best to transform (1) to a uniformly rotating frame as in Section VI. It follows from the description of the nonlinear system in this reference frame that Arnold diffusion is expected for $\delta>0$, even for sufficiently small $\epsilon$. Numerical experiments described in Section VII tend to corroborate the conjecture that gravitational ionization is tantamount to Arnold diffusion in this system. For background material, this paper relies heavily on our previous detailed treatment of the nonlinear problem for the case where the incident wave is essentially a simple monochromatic Fourier componentt; however, we have attempted to present sufficient detail here in order to render the present paper essentially self-contained.

\section{HAMILTONIAN DESCRIPTION IN DELAUNAY ELEMENTS}

Using (11), (2) and (4), we can write the associated Hamiltonian for this system as the sum of the Kepler Hamiltonian and the quadrupole perturbation given by $\frac{1}{2} \epsilon \mathcal{K}_{i j} x^{i} x^{j}$. The motion is taken to be in the $(x, y)$-plane, since the radiation is transverse and normally incident on the orbital plane; therefore, polar coordinates are convenient. Defining $\phi(t)$ and $\psi(t)$ as

$$
\begin{aligned}
& \phi(t)=\Omega^{2}\left\{\cos 2 \Omega t+\frac{\delta}{2}[(\alpha-\beta) \cos \Omega t+(\alpha+\beta) \cos 3 \Omega t]\right\}, \\
& \psi(t)=\Omega^{2}\left\{\sin 2 \Omega t+\frac{\delta}{2}[(\alpha-\beta) \sin \Omega t+(\alpha+\beta) \sin 3 \Omega t]\right\},
\end{aligned}
$$

the Hamiltonian in polar coordinates may be written as

$$
\mathcal{H}=\frac{1}{2}\left(p_{r}^{2}+\frac{p_{\theta}^{2}}{r^{2}}\right)-\frac{k}{r}+\epsilon r^{2}[\phi(t) \cos 2 \theta+\psi(t) \sin 2 \theta] .
$$

To express the Hamiltonian in a form particularly suitable for the analysis of orbital dynamics, we transform to the Delaunay elements $(L, G, \ell, g)$. To this end, consider the bounded motion of the test particle according to the Hamiltonian (6). At each instant of time, the particle can be described as belonging to an osculating Keplerian ellipse; that is, the perturbed motion passes through an infinite sequence of osculating ellipses in the course of time. Each such ellipse is described by the unperturbed Hamiltonian

$$
H=\frac{1}{2}\left(p_{r}^{2}+\frac{p_{\theta}^{2}}{r^{2}}\right)-\frac{k}{r}
$$

where we consider only bounded motions with $E=H\left(p_{r}, p_{\theta}, r, \theta\right)<0$. Let us now consider the canonical transformation from $\left(p_{r}, p_{\theta}, r, \theta\right)$ to variables intrinsic to the ellipse. Thus we define action variables

$$
L:=\left(\frac{-k^{2}}{2 E}\right)^{\frac{1}{2}}, \text { and } \quad G:=p_{\theta},
$$

which correspond to an osculating Keplerian ellipse with semimajor axis $a, a=L^{2} / k$, and eccentricity $e$,

$$
e=\left(1-\frac{G^{2}}{L^{2}}\right)^{\frac{1}{2}}
$$

such that $0 \leq e<1$. The equation of this ellipse is given by

$$
r=a(1-e \cos \widehat{u}), \quad \text { or } \quad r=a \frac{1-e^{2}}{1+e \cos v},
$$

where $\widehat{u}$ is the eccentric anomaly and $v$ is the true anomaly. The new canonical angle variables $\ell$ and $g$ are then given by 


$$
\ell=\widehat{u}-e \sin \widehat{u}, \quad g=\theta-v .
$$

In the following, we exclude $e=0$ and focus attention instead on noncircular elliptical orbits. The resulting Hamiltonian of the perturbed system in Delaunay variables is

$$
\mathcal{H}=-\frac{k^{2}}{2 L^{2}}+\epsilon[\mathcal{C}(L, G, \ell, g) \phi(t)+\mathcal{S}(L, G, \ell, g) \psi(t)],
$$

where, in polar coordinates, $\mathcal{C}=r^{2} \cos 2 \theta$ and $\mathcal{S}=r^{2} \sin 2 \theta$. In Delaunay elements, $\mathcal{C}(L, G, \ell, g)$ and $\mathcal{S}(L, G, \ell, g)$ are given by

$$
\begin{aligned}
\mathcal{C}(L, G, \ell, g)= & \frac{5}{2} a^{2} e^{2} \cos 2 g \\
& +a^{2} \sum_{\nu=1}^{\infty}\left(A_{\nu}(e) \cos 2 g \cos \nu \ell-B_{\nu}(e) \sin 2 g \sin \nu \ell\right), \\
\mathcal{S}(L, G, \ell, g)=\frac{5}{2} a^{2} e^{2} \sin 2 g & \\
& +a^{2} \sum_{\nu=1}^{\infty}\left(A_{\nu}(e) \sin 2 g \cos \nu \ell+B_{\nu}(e) \cos 2 g \sin \nu \ell\right),
\end{aligned}
$$

where

$$
\begin{aligned}
& A_{\nu}(e)=\frac{4}{\nu^{2} e^{2}}\left(2 \nu e\left(1-e^{2}\right) J_{\nu}^{\prime}(\nu e)-\left(2-e^{2}\right) J_{\nu}(\nu e)\right), \\
& B_{\nu}(e)=-\frac{8}{\nu^{2} e^{2}} \sqrt{1-e^{2}}\left(e J_{\nu}^{\prime}(\nu e)-\nu\left(1-e^{2}\right) J_{\nu}(\nu e)\right) .
\end{aligned}
$$

Here $J_{\nu}$ is the Bessel function of order $\nu$, and a prime indicates the derivative of the function with respect to its argument. The dynamical equations are derived in the usual way from the Hamiltonian (7). Moreover, these equations are given in Delaunay elements by

$$
\begin{aligned}
\dot{L} & =-\epsilon\left(\frac{\partial \mathcal{C}(L, G, \ell, g)}{\partial \ell} \phi(t)+\frac{\partial \mathcal{S}(L, G, \ell, g)}{\partial \ell} \psi(t)\right), \\
\dot{G} & =-\epsilon\left(\frac{\partial \mathcal{C}(L, G, \ell, g)}{\partial g} \phi(t)+\frac{\partial \mathcal{S}(L, G, \ell, g)}{\partial g} \psi(t)\right), \\
\dot{\ell} & =\omega+\epsilon\left(\frac{\partial \mathcal{C}(L, G, \ell, g)}{\partial L} \phi(t)+\frac{\partial \mathcal{S}(L, G, \ell, g)}{\partial L} \psi(t)\right), \\
\dot{g} & =\epsilon\left(\frac{\partial \mathcal{C}(L, G, \ell, g)}{\partial G} \phi(t)+\frac{\partial \mathcal{S}(L, G, \ell, g)}{\partial G} \psi(t)\right),
\end{aligned}
$$

where $\omega$ is the Keplerian frequency of the binary given by $\omega^{2}=k / a^{3}$.

\section{BIFURCATION FUNCTION}

To establish the continuation (persistence) of periodic orbits of the Kepler problem to the system (10), we employ a method proposed in Ref. 2 and used in Ref. 1; we only state the main ideas here and the reader is referred to these references for details.

System (10) has the abstract form

$$
\dot{u}=F(u)+\epsilon h(u, t),
$$

where $u$ is a coordinate on a manifold $M$ that consists of a cross product of Euclidean spaces and tori, and the function $h$ is periodic with period $2 \pi / \Omega$ in its second argument. We consider solutions $t \mapsto u(t, \xi, \epsilon)$ of (11) with initial condition $u(0, \xi, \epsilon)=\xi, \xi \in M$, and define the $m$ th order Poincaré map by $\mathcal{P}^{m}(\xi, \epsilon)=u(2 \pi m / \Omega, \xi, \epsilon)$. Fixed

points of this $m$ th order Poincaré map correspond to periodic solutions of (11). Consider the unperturbed periodic solutions of (11) with $\epsilon=0$. These correspond to fixed points of the unperturbed $m$ th order Poincaré map defined 
by $p^{m}(\xi)=\mathcal{P}^{m}(\xi, 0)$. Let us now suppose that there is a submanifold $\mathcal{Z} \subset M$ that consists of fixed points of $p^{m}$, and $\zeta \in \mathcal{Z}$. If there is a continuous curve $\epsilon \mapsto \kappa(\epsilon)$ in $M$ such that $\kappa(0)=\zeta$ and $\mathcal{P}^{m}(\kappa(\epsilon), \epsilon) \equiv \kappa(\epsilon)$, i.e. for each fixed value of $\epsilon, \kappa(\epsilon)$ is a fixed point of the $m$ th order Poincaré map, then the unperturbed periodic orbit is continuable. The continuation is established by the method of Lyapunov-Schmidt reduction to the Implicit Function Theorem and requires that every vector in $T_{\zeta} M$ that is tangent to the submanifold $\mathcal{Z}$ be in the kernel of the infinitesimal displacement $\mathcal{D}(\zeta)=D p^{m}(\zeta)-I$. This is equivalent to the requirement that for each $\zeta \in \mathcal{Z}$, the dimension of the kernel of the infinitesimal displacement at $\zeta$ be equal to the dimension of the manifold $\mathcal{Z}$. The manifold $\mathcal{Z}$ is called normally nondegenerate if it satisfies this condition.

Let $\mathcal{Z}$ be a normally nondegenerate submanifold of $M$ with dimension $q$; then, the range of the infinitesimal displacement at each point of $\mathcal{Z}$ has codimension $q$. For each $\zeta \in \mathcal{Z}$, there is a vector complement $\widehat{\mathcal{S}}(\zeta)$ to the range of the infinitesimal displacement of dimension $q$. We denote the projection of $T_{\zeta} M$ to $\widehat{\mathcal{S}}(\zeta)$ by $\widehat{s}(\zeta)$.

Let $\zeta \in \mathcal{Z}$ and consider the curve in the manifold $M$ defined by $\epsilon \mapsto \mathcal{P}^{m}(\zeta, \epsilon)$; it passes through $\zeta$ at $\epsilon=0$ and its tangent vector at $\epsilon=0$ is in $T_{\zeta} M$. This tangent vector can be identified with the partial derivative $\mathcal{P}_{\epsilon}^{m}(\zeta, 0)$. The bifurcation function $\mathcal{B}$ is defined to be the map from $\mathcal{Z}$ to the complement $\widehat{\mathcal{S}}$ of the range of the infinitesimal displacement

$$
\mathcal{B}(\zeta)=\widehat{s}(\zeta) \mathcal{P}_{\epsilon}^{m}(\zeta, 0)
$$

so that in local coordinates $\mathcal{B}: \mathbb{R}^{q} \rightarrow \mathbb{R}^{q}$. We define $\zeta \in \mathcal{Z}$ to be a simple zero of the bifurcation function if $\mathcal{B}(\zeta)=0$ and the derivative $D \mathcal{B}(\zeta)$ is invertible. The following continuation theorem is proved in Ref. 2 .

Theorem III.1 Let $\mathcal{Z}$ denote a normally nondegenerate fixed point submanifold of $M$ for the system (11). If $\zeta \in \mathcal{Z}$ is a simple zero of the corresponding bifurcation function, then the unperturbed periodic orbit of (11) with initial point $\zeta$ is continuable.

To apply Theorem III.1 to the perturbed Kepler problem, we must compute the partial derivative $\mathcal{P}_{\epsilon}^{m}(\zeta, 0)$ of the corresponding Poincaré map. For the system (10), the manifold $M$ is the four dimensional Delaunay coordinate space and the Poincaré map is defined as the strobe with period $2 \pi m / \Omega$ where $\Omega$ is the frequency of the perturbation. The partial derivative is obtained from the solution $t \mapsto W(t)$ of the second variational initial value problem

$$
\dot{W}=D F(u(t, \zeta, 0)) W+h(u(t, \zeta, 0), t), \quad W(0)=0 .
$$

In fact, we have $W(t)=u_{\epsilon}(t, \zeta, 0)$ and

$$
\mathcal{P}_{\epsilon}^{m}(\zeta, 0)=W(2 \pi m / \Omega) .
$$

We use Theorem [II.1 to establish the existence of periodic orbits for the system (10). Of course, this will establish the existence of periodic orbits for our model system (i]), which describes the perturbation of a Keplerian binary system by a multi-frequency periodic gravitational wave. We begin by identifying the normally nondegenerate fixed point submanifold of $M$ mentioned in Theorem III.1. Recall that the frequency of the unperturbed periodic Keplerian orbit is $\omega=k^{2} / L^{3}$. The three-dimensional manifold

$$
\mathcal{Z}^{L}:=\{(L, G, \ell, g): m \omega=n \Omega\}
$$

where $m$ and $n$ are relatively prime positive integers, is a normally nondegenerate submanifold of $M$ (cf. Ref. 1). Furthermore, the range of the infinitesimal displacement is complemented by the span of the vectors

$$
\left(\begin{array}{l}
1 \\
0 \\
0 \\
0
\end{array}\right), \quad\left(\begin{array}{l}
0 \\
1 \\
0 \\
0
\end{array}\right), \quad\left(\begin{array}{l}
0 \\
0 \\
0 \\
1
\end{array}\right) .
$$

The bifurcation function associated with (10) is the projection of the partial derivative $\mathcal{P}_{\epsilon}^{m}(L, G, \ell, g, 0)$ on the manifold $\mathcal{Z}^{L}$ onto the complement of the range of the infinitesimal displacement. To determine the bifurcation function, we solve the variational initial value problem

$$
\begin{aligned}
\dot{L}_{\epsilon} & =-\frac{\partial \mathcal{C}}{\partial \ell}(L, G, \ell+\omega t, g) \phi(t)-\frac{\partial \mathcal{S}}{\partial \ell}(L, G, \ell+\omega t, g) \psi(t), \\
\dot{G}_{\epsilon} & =-\frac{\partial \mathcal{C}}{\partial g}(L, G, \ell+\omega t, g) \phi(t)-\frac{\partial \mathcal{S}}{\partial g}(L, G, \ell+\omega t, g) \psi(t), \\
\dot{\ell}_{\epsilon} & =-\frac{3 k^{2}}{L^{4}} L_{\epsilon}+\frac{\partial \mathcal{C}}{\partial L}(L, G, \ell+\omega t, g) \phi(t)+\frac{\partial \mathcal{S}}{\partial L}(L, G, \ell+\omega t, g) \psi(t), \\
\dot{g}_{\epsilon} & =\frac{\partial \mathcal{C}}{\partial G}(L, G, \ell+\omega t, g) \phi(t)+\frac{\partial \mathcal{S}}{\partial G}(L, G, \ell+\omega t, g) \psi(t)
\end{aligned}
$$


with zero initial values; then, the solution evaluated at $t=m(2 \pi / \Omega)$ is projected to the complement of the range of the infinitesimal displacement. It follows from a detailed analysis that one can set the initial value of time equal to zero, as we have done, with no loss in generality. The bifurcation function is thus given by

$$
\mathcal{B}(G, \ell, g)=\left(B^{L}(G, \ell, g), B^{G}(G, \ell, g), B^{g}(G, \ell, g)\right),
$$

where

$$
B^{L}(G, \ell, g):=-\frac{\partial \mathcal{I}}{\partial \ell}, \quad B^{G}(G, \ell, g):=-\frac{\partial \mathcal{I}}{\partial g}, \quad B^{g}(G, \ell, g):=\frac{\partial \mathcal{I}}{\partial G}
$$

and

$$
\mathcal{I}:=\int_{0}^{2 \pi m / \Omega}[\mathcal{C}(L, G, \ell+\omega t, g) \phi(t)+\mathcal{S}(L, G, \ell+\omega t, g) \psi(t)] d t .
$$

To evaluate $\mathcal{I}$, we use the resonance relation $n \Omega=m \omega$ to change the variable of integration from $t$ to $\widehat{\sigma}=\Omega t / m+\ell / n$, then observe that the integrand of $\mathcal{I}$ is periodic with period $2 \pi$ and substitute the Fourier series expansion for $\mathcal{C}$ and $\mathcal{S}$. After performing these steps, we obtain the following expression for $\mathcal{I}$ in case $n=1$

$$
\begin{gathered}
\mathcal{I}=\pi m a^{2} \Omega\left\{U_{2 m}(e) \cos (2 g+2 m \ell)+\frac{\delta}{2}\left[(\alpha-\beta) U_{m}(e) \cos (2 g+m \ell)\right.\right. \\
\left.\left.+(\alpha+\beta) U_{3 m}(e) \cos (2 g+3 m \ell)\right]\right\}
\end{gathered}
$$

where

$$
U_{\nu}(e)=A_{\nu}(e)+B_{\nu}(e)
$$

If $n=2$ and $m$ is odd, then

$$
\mathcal{I}=\pi m a^{2} \Omega U_{m}(e) \cos (2 g+m \ell) ;
$$

while for $n=3$, if $m$ is prime relative to 3 , then

$$
\mathcal{I}=\frac{1}{2} \pi m a^{2} \Omega \delta(\alpha+\beta) U_{m}(e) \cos (2 g+m \ell) .
$$

It turns out that $\mathcal{I}=0$ for $n>3$, as expected.

We show in Ref. 1 that the bifurcation function does not have a simple zero for an incident monochromatic gravitational wave of definite helicity. The role of the secondary components of the wave is to resolve this issue for the bifurcation problem under consideration here. This is indeed the case for $n=1$ as demonstrated in the next section. However, for $n=2$ and $n=3$ simple zeros do not exist, and the consideration of these cases would require the calculation of the solutions of higher order variational initial value problems. On the other hand, higher order perturbing functions of order $\epsilon^{2}$, etc., are neglected in the formulation of equation (11), which is the starting point of our analysis. It follows from this remark that the treatment of the cases $n=2$ and $n=3$ is beyond the scope of this work. Thus, we assume $n=1$ in the remainder of this section.

Substituting (13) into (12), we obtain the following explicit form for the bifurcation function $(n=1)$

$$
\begin{aligned}
B^{L}(G, \ell, g)=2 \pi m^{2} a^{2} \Omega\left\{U_{2 m}(e) \sin (2 g+2 m \ell)\right. & \\
+ & \frac{\delta}{4}\left[(\alpha-\beta) U_{m}(e) \sin (2 g+m \ell)\right. \\
& \left.\left.+3(\alpha+\beta) U_{3 m}(e) \sin (2 g+3 m \ell)\right]\right\}, \\
B^{G}(G, \ell, g)=2 \pi m a^{2} \Omega\left\{U_{2 m}(e) \sin (2 g+2 m \ell)\right. & \\
+ & \frac{\delta}{2}\left[(\alpha-\beta) U_{m}(e) \sin (2 g+m \ell)\right. \\
& \left.\left.+(\alpha+\beta) U_{3 m}(e) \sin (2 g+3 m \ell)\right]\right\} \\
B^{g}(G, \ell, g)=-\pi m a^{2} \Omega\left(\frac{G}{e L^{2}}\right)\left\{U_{2 m}^{\prime}(e) \cos (2 g+2 m \ell)\right. & \\
+ & \frac{\delta}{2}\left[(\alpha-\beta) U_{m}^{\prime}(e) \cos (2 g+m \ell)\right. \\
+ & \left.\left.(\alpha+\beta) U_{3 m}^{\prime}(e) \cos (2 g+3 m \ell)\right]\right\}
\end{aligned}
$$


where in the expression for $B^{g}$ we have used the fact that the eccentricity $e$ and the Delaunay element $G$ are related by $G= \pm L \sqrt{1-e^{2}}$. We therefore make a change of variable from $G$ to $e$ and observe that the zeros and their multiplicities for the bifurcation function $\mathcal{B}$ are identical to those of the function

$$
\mathcal{F}(e, \ell, g)=\left(F^{L}(e, \ell, g), F^{G}(e, \ell, g), F^{g}(e, \ell, g)\right),
$$

where

$$
\begin{array}{r}
F^{L}(e, \ell, g)=U_{2 m}(e) \sin (2 g+2 m \ell) \\
+\frac{\delta}{4}\left[(\alpha-\beta) U_{m}(e) \sin (2 g+m \ell)\right. \\
\left.+3(\alpha+\beta) U_{3 m}(e) \sin (2 g+3 m \ell)\right], \\
F^{G}(e, \ell, g)=U_{2 m}(e) \sin (2 g+2 m \ell) \\
+\frac{\delta}{2}\left[(\alpha-\beta) U_{m}(e) \sin (2 g+m \ell)\right. \\
\left.\quad+(\alpha+\beta) U_{3 m}(e) \sin (2 g+3 m \ell)\right], \\
F^{g}(e, \ell, g)=U_{2 m}^{\prime}(e) \cos (2 g+2 m \ell) \\
+\frac{\delta}{2}\left[(\alpha-\beta) U_{m}^{\prime}(e) \cos (2 g+m \ell)\right. \\
\left.+(\alpha+\beta) U_{3 m}^{\prime}(e) \cos (2 g+3 m \ell)\right] .
\end{array}
$$

To apply Theorem [II.1, we must determine the simple zeros of the bifurcation function (14) or, equivalently, (15). We will show that simple zeros exist by a perturbation argument which is presented in the next section.

\section{ZEROS OF A DEGENERATE BIFURCATION FUNCTION}

The bifurcation function (15) has the following abstract form

$$
\Delta(\mu, \widehat{\epsilon})=\tau(\mu)+\widehat{\epsilon} \varphi(\mu)=0, \quad \mu \in \mathbb{R}^{3},
$$

where the functions $\Delta: \mathbb{R}^{3} \times \mathbb{R} \mapsto \mathbb{R}^{3}$ and $\tau, \varphi: \mathbb{R}^{3} \mapsto \mathbb{R}^{3}$ are given in components by

$$
\tau(\mu)=\left(\begin{array}{c}
\tau_{1}(\mu) \\
\tau_{2}(\mu) \\
\tau_{3}(\mu)
\end{array}\right), \quad \varphi(\mu)=\left(\begin{array}{c}
\varphi_{1}(\mu) \\
\varphi_{2}(\mu) \\
\varphi_{3}(\mu)
\end{array}\right),
$$

and where the first two components of $\tau$ are equal. In fact, we define $\widehat{\tau}=\tau_{1}=\tau_{2}$.

Remark IV.1 The fact that the bifurcation function has the abstract form (16) is not accidental. Every normally incident gravitational plane wave whose Fourier representation has as its dominant term a purely circularly polarized wave will result in a bifurcation function of the form (10) at resonance.

We will determine the zero set for $\Delta$ in case $\widehat{\epsilon}$ is sufficiently small.

Lemma IV.2 Suppose $\Delta$ is defined by (10). If $\eta \in \mathbb{R}^{3}$ is such that $\tau(\eta)=0$ while the vectors $\operatorname{grad} \widehat{\tau}(\eta)$ and $\operatorname{grad} \tau_{3}(\eta)$ are linearly independent, then there is a curve $s \mapsto \Gamma(s)$ in $\mathbb{R}^{3}$ such that $\Gamma(0)=\eta, \Gamma^{\prime}(0) \neq 0$, and $\Delta(\Gamma(s), 0) \equiv 0$. If such a curve exists and $s=0$ is a simple zero of the real-valued function $s \mapsto \varphi_{2}(\Gamma(s))-\varphi_{1}(\Gamma(s))$, then there is a curve $\widehat{\epsilon} \mapsto \Upsilon(\widehat{\epsilon})$ in $\mathbb{R}^{3}$ such that $\Upsilon(0)=\eta$ and $\Delta(\Upsilon(\widehat{\epsilon}), \widehat{\epsilon}) \equiv 0$. Moreover, for each sufficiently small $\widehat{\epsilon} \neq 0$, the point $\Upsilon(\widehat{\epsilon}) \in \mathbb{R}^{3}$ is a simple zero of the function $\mu \mapsto \Delta(\mu, \widehat{\epsilon})$.

Proof. The linearly independent vectors $\operatorname{grad} \widehat{\tau}(\eta)$ and $\operatorname{grad} \tau_{3}(\eta)$ span a plane $Q$ in $\mathbb{R}^{3}$. Let $S$ denote one of the two possible rotation operators in space that preserves $Q$ and rotates each vector in $Q$ through $\pi / 2$ radians. We note that $S \operatorname{grad} \widehat{\tau}(\eta)$ and $S \operatorname{grad} \tau_{3}(\eta)$ are linearly independent vectors in $Q$. Also, we let $v$ denote a vector in space such that the set $\left\{S \operatorname{grad} \widehat{\tau}(\eta), S \operatorname{grad} \tau_{3}(\eta), v\right\}$ is linearly independent. This requirement is satisfied, for instance, if we choose $v$ to be equal to the cross product of $S \operatorname{grad} \widehat{\tau}(\eta)$ and $S \operatorname{grad} \tau_{3}(\eta)$.

The function $w: \mathbb{R}^{3} \mapsto \mathbb{R}^{3}$ defined by

$$
\left(\mu_{1}, \mu_{2}, \mu_{3}\right) \mapsto \mu_{1} S \operatorname{grad} \tau_{3}(\eta)+\mu_{2} S \operatorname{grad} \widehat{\tau}(\eta)+\mu_{3} v+\eta
$$


is invertible. We use it to define $\Theta: \mathbb{R}^{3} \rightarrow \mathbb{R}^{2}$ given by

$$
\Theta\left(\mu_{1}, \mu_{2}, \mu_{3}\right)=\left(\widehat{\tau}\left(w\left(\mu_{1}, \mu_{2}, \mu_{3}\right)\right), \tau_{3}\left(w\left(\mu_{1}, \mu_{2}, \mu_{3}\right)\right) .\right.
$$

It follows that $\Theta(0)=0$. Moreover, the derivative of the transformation

$$
\left(\mu_{1}, \mu_{2}\right) \mapsto\left(\widehat{\tau}\left(w\left(\mu_{1}, \mu_{2}, 0\right)\right), \tau_{3}\left(w\left(\mu_{1}, \mu_{2}, 0\right)\right)\right.
$$

at $\left(\mu_{1}, \mu_{2}\right)=(0,0)$ is given by the matrix

$$
\left(\begin{array}{cc}
\operatorname{grad} \widehat{\tau}(\eta) \cdot S \operatorname{grad} \tau_{3}(\eta) & 0 \\
0 & \operatorname{grad} \tau_{3}(\eta) \cdot S \operatorname{grad} \widehat{\tau}(\eta)
\end{array}\right)
$$

that represents the partial derivative of $\Theta$ with respect to its first two arguments at the origin.

Using the linear independence of $\operatorname{grad} \widehat{\tau}(\eta)$ and $\operatorname{grad} \tau_{3}(\eta)$, it is easy to see that the diagonal elements of the matrix (17) are both nonzero. Thus, by the Implicit Function Theorem, there is a unique curve $s \mapsto \sigma(s)$ in the $\left(\mu_{1}, \mu_{2}\right)$-plane such that $\sigma(0)=(0,0)$ and $\Theta(\sigma(s), s) \equiv 0$. The curve $\Gamma(s):=w(\sigma(s), s)$ is such that $\Gamma(0)=\eta$ and $\Delta(\Gamma(s), 0) \equiv 0$. Moreover,

$$
\Gamma^{\prime}(0)=D w(0)\left(\begin{array}{c}
\sigma^{\prime}(0) \\
1
\end{array}\right)
$$

Since $D w(0)$ is invertible and the vector $\left(\sigma^{\prime}(0), 1\right) \neq 0$, we have that $\Gamma^{\prime}(0) \neq 0$. This proves the first assertion of the theorem.

Under the assumption that $s=0$ is a simple zero of the function $s \mapsto \varphi_{2}(\Gamma(s))-\varphi_{1}(\Gamma(s))$, we will use the LyapunovSchmidt reduction procedure to show that the corresponding point $\eta=\Gamma(0)$ in $\mathbb{R}^{3}$ is continuable as a zero of $\Delta$. As $\Gamma^{\prime}(0) \neq 0$, the image $\widehat{\mathcal{Z}}$ of $\Gamma$ is locally a one dimensional submanifold of $\mathbb{R}^{3}$. Moreover, $\widehat{\mathcal{Z}}$ is normally nondegenerate in the sense that at each point $\widehat{z} \in \widehat{\mathcal{Z}}$ sufficiently close to $\eta$, the kernel $\widehat{K}$ of $D \tau(\widehat{z})$ is exactly the one dimensional tangent space of $\widehat{\mathcal{Z}}$. In fact, since this tangent space is clearly in $\widehat{K}$, it suffices to show that $\widehat{K}$ is one dimensional. The derivative of $\tau$ is expressed in the standard vector notation by

$$
D \tau(\widehat{z})=\left(\begin{array}{l}
\operatorname{grad} \widehat{\tau}(\widehat{z}) \\
\operatorname{grad} \widehat{\tau}(\widehat{z}) \\
\operatorname{grad} \tau_{3}(\widehat{z})
\end{array}\right) .
$$

Since the vectors $\operatorname{grad} \widehat{\tau}(\widehat{z})$ and $\operatorname{grad} \tau_{3}(\widehat{z})$ are linearly independent at $\widehat{z}=\eta$, they will, by continuity, remain linearly independent in an open neighborhood of $\eta$. Thus, their span is two dimensional at each point of this neighborhood. In particular, for $\widehat{z}$ in this neighborhood, it is clear (by matrix multiplication) that $\operatorname{grad} \widehat{\tau}(\widehat{z})$ and $\operatorname{grad} \tau_{3}(\widehat{z}) \operatorname{are~not}$ both in $\widehat{K}$. This proves that $\widehat{K}$ is one dimensional. It follows from the same Lyapunov-Schmidt reduction procedure that is used to prove Theorem [II.1 (see Ref. 2) that if $\Pi(\widehat{z})$ denotes a projection to the complement $\widehat{\mathcal{S}}$ of the range of $D \tau(\widehat{z})$, then simple zeros of the map from $\widehat{\mathcal{Z}}$ to $\mathbb{R}$ given by $\widehat{z} \mapsto \Pi(\widehat{z}) \Delta_{\hat{\epsilon}}(\widehat{z}, 0)$ are continuable. Thus, if $\eta$ is such a simple zero, there is a curve $\widehat{\epsilon} \mapsto \Upsilon(\widehat{\epsilon})$ such that $\Upsilon(0)=\eta$ and $\Delta(\Upsilon(\widehat{\epsilon}), \widehat{\epsilon}) \equiv 0$ as required in the lemma.

To construct such a projection into $\widehat{\mathcal{S}}$, it can be shown that, in fact, the range of $D \tau(\widehat{z})$ is spanned by the transpositions of the vectors $(1,1,0)$ and $(0,0,1)$. To see this, it is sufficient to note that $D \tau(\widehat{z}) S \operatorname{grad} \widehat{\tau}(\widehat{z})$ is the transpose of a scalar multiple of $(0,0,1)$ while $D \tau(\widehat{z}) S \operatorname{grad} \tau_{3}(\widehat{z})$ is a scalar multiple of the transpose of $(1,1,0)$. The transpose of the vector $(0,1,0)$ clearly spans a complement to the range that we will denote by $\widehat{\mathcal{S}}$. Thus, if $\left(\rho_{1}, \rho_{2}, \rho_{3}\right) \in \mathbb{R}^{3}$, the projection $\Pi$ is easily computed and is given by $\Pi\left(\rho_{1}, \rho_{2}, \rho_{3}\right)=\rho_{2}-\rho_{1}$. Note that $\Pi$ does not depend on the base point $\widehat{z} \in \widehat{\mathcal{Z}}$.

Using the projection $\Pi$ and the definition of $\Delta$, the Lyapunov-Schmidt reduced function $\widehat{z} \mapsto \Pi(\widehat{z}) \Delta_{\hat{\epsilon}}(\widehat{z}, 0)$ is given by $\widehat{z} \mapsto \varphi_{2}(\widehat{z})-\varphi_{1}(\widehat{z})$ for $\widehat{z} \in \widehat{\mathcal{Z}}$. Thus, if, in the coordinates of $\widehat{\mathcal{Z}}, s=0$ is a simple zero of $s \mapsto \varphi_{2}(\Gamma(s))-\varphi_{1}(\Gamma(s))$, then $\eta=\Gamma(0)$ is a continuable zero of $\Delta$ given by a curve $\Upsilon$, as required in the statement of the lemma.

It remains to show that $\Upsilon(\widehat{\epsilon})$, for sufficiently small $\widehat{\epsilon} \neq 0$, is in fact a simple zero of the function $\mu \mapsto \Delta(\mu, \widehat{\epsilon})$. To this end, it suffices to show that the matrix $D \Delta(\Upsilon(\widehat{\epsilon}), \widehat{\epsilon})$ does not have a zero eigenvalue. But, under the hypotheses of the lemma, the matrix $D \Delta(\eta, 0)$ has a one dimensional kernel, namely the tangent space of $\widehat{\mathcal{Z}}$. This means that $D \Delta(\eta, 0)$ has exactly one zero eigenvalue. By the continuity of eigenvalues of matrices, there is a smooth family of eigenvalues $\lambda(\widehat{\epsilon})$ such that $\lambda(0)=0$ and a corresponding smooth family of eigenvectors $V(\widehat{\epsilon})$ such that $V(0)$ is a nonzero vector tangent to $\widehat{\mathcal{Z}}$ with 


$$
D \Delta(\Upsilon(\widehat{\epsilon}), \widehat{\epsilon}) V(\widehat{\epsilon})=\lambda(\widehat{\epsilon}) V(\widehat{\epsilon}) .
$$

It suffices to show that if $\widehat{\epsilon}$ is positive and sufficiently small, then $\lambda(\widehat{\epsilon}) \neq 0$. By continuity, the remaining two eigenvalues of the matrix will be nonzero as well. The desired result follows as soon as we show that the derivative $\lambda^{\prime}(0) \neq 0$. For this we have

$$
D^{2} \Delta(\eta, 0)\left(\Upsilon^{\prime}(0), V(0)\right)+D \Delta_{\hat{\epsilon}}(\eta, 0) V(0)+D \Delta(\eta, 0) V^{\prime}(0)=\lambda^{\prime}(0) V(0),
$$

where $D$ denotes differentiation with respect to the space variable $\mu \in \mathbb{R}^{3}$. After projection by $\Pi$ into the complement of the range of $D \Delta(\eta, 0)$,

$$
\Pi D^{2} \Delta(\eta, 0)\left(\Upsilon^{\prime}(0), V(0)\right)+\Pi D \Delta_{\hat{\epsilon}}(\eta, 0) V(0)=\lambda^{\prime}(0) \Pi V(0) .
$$

We claim that $\Pi D^{2} \Delta(\eta, 0)\left(\Upsilon^{\prime}(0), V(0)\right)=0$. Once this claim is proved, the fact that $\lambda^{\prime}(0) \neq 0$ would follow provided $\Pi D \Delta_{\hat{\epsilon}}(\eta, 0) V(0) \neq 0$. The key point to note is that the projection operator, $\Pi$, is independent of the base point, i.e. the range of the projection operator is spanned by a constant basis vector independent of the base point. Thus since $\Delta_{\hat{\epsilon}}(\Gamma(s), 0)=\varphi(\Gamma(s))$, we have

$$
\left.\frac{d}{d s} \Pi \varphi(\Gamma(s))\right|_{s=0}=\Pi D \varphi(\eta) \Gamma^{\prime}(0)=\Pi D \Delta_{\hat{\epsilon}}(\eta, 0) \Gamma^{\prime}(0) .
$$

Since $\widehat{\mathcal{Z}}$ is one dimensional, $\Gamma^{\prime}(0)$ is just a scalar multiple of $V(0)$ and therefore if $\Pi D \varphi(\eta) \Gamma^{\prime}(0) \neq 0$, then $\Pi D \Delta_{\hat{\epsilon}}(\eta, 0) V(0) \neq 0$. By the definition of the components of $\varphi$ and of the projection $\Pi$, we have

$$
\left.\frac{d}{d s} \Pi \varphi(\Gamma(s))\right|_{s=0}=\left.\frac{d}{d s}\left[\varphi_{2}(\Gamma(s))-\varphi_{1}(\Gamma(s))\right]\right|_{s=0} .
$$

Thus, if $s=0$ is a simple zero of $s \mapsto \varphi_{2}(\Gamma(s))-\varphi_{1}(\Gamma(s))$, then

$$
\Pi D \Delta_{\hat{\epsilon}}(\eta, 0) V(0) \neq 0,
$$

as required.

To verify the claim, note that since $\Pi$ projects to the complement of the range of $D \Delta(\eta, 0)$ and since $\Pi$ does not depend on the base point on $\widehat{\mathcal{Z}}$, we have $\Pi D \Delta(\Gamma(\vartheta), 0) \Upsilon^{\prime}(\vartheta) \equiv 0$ for the real variable $\vartheta$ in the common domain of $\Gamma$ and $\Upsilon$. This implies that

$$
\begin{aligned}
0 & =\left.\frac{d}{d \vartheta}\left[\Pi D \Delta(\Gamma(\vartheta), 0) \Upsilon^{\prime}(\vartheta)\right]\right|_{\vartheta=0} \\
& =\Pi D^{2} \Delta(\eta, 0)\left(\Gamma^{\prime}(0), \Upsilon^{\prime}(0)\right)+\Pi D \Delta(\eta, 0) \Upsilon^{\prime \prime}(0) \\
& =\widehat{c} \Pi D^{2} \Delta(\eta, 0)\left(V(0), \Upsilon^{\prime}(0)\right),
\end{aligned}
$$

where $\widehat{c} V(0)=\Gamma^{\prime}(0)$. Since $\widehat{c} \neq 0$, the lemma is proved.

\section{PERIODIC ORBITS}

We now use Lemma IV.2 to prove that some of the zeros of the bifurcation function (15) are simple. We note that once this result is established, it will follow from Theorem III.1 that the corresponding bounded orbits of the Keplerian two-body system are continuable under perturbation by periodic gravitational waves.

If $\delta=0$, then the zeros of (15) are the union of the following one dimensional sets

$$
\begin{array}{ll}
\mathcal{Z}_{1}^{+}=\{(e, \ell, g): 2 g+2 m \ell=0, & \left.U_{2 m}^{\prime}(e)=0\right\}, \\
\mathcal{Z}_{1}^{-}=\{(e, \ell, g): 2 g+2 m \ell=\pi, & \left.U_{2 m}^{\prime}(e)=0\right\}, \\
\mathcal{Z}_{2}^{+}=\left\{(e, \ell, g): 2 g+2 m \ell=\frac{\pi}{2},\right. & \left.U_{2 m}(e)=0\right\}, \\
\mathcal{Z}_{2}^{-}=\left\{(e, \ell, g): 2 g+2 m \ell=\frac{3 \pi}{2},\right. & \left.U_{2 m}(e)=0\right\} .
\end{array}
$$


We will show that the zeros in the sets $\mathcal{Z}_{1}^{ \pm}$and $\mathcal{Z}_{2}^{ \pm}$continue to simple zeros of the bifurcation function for sufficiently small $\delta \neq 0$. To conform with Lemma IV.2, we use (15) to identify the components of $\Delta$ as they appear in the lemma as follows:

$$
\begin{aligned}
\widehat{\tau}(e, \ell, g) & =U_{2 m}(e) \sin (2 g+2 m \ell), \\
\tau_{3}(e, \ell, g) & =U_{2 m}^{\prime}(e) \cos (2 g+2 m \ell), \\
\varphi_{1}(e, \ell, g)= & \frac{1}{4}\left[(\alpha-\beta) U_{m}(e) \sin (2 g+m \ell)\right. \\
& \left.+3(\alpha+\beta) U_{3 m}(e) \sin (2 g+3 m \ell)\right], \\
\varphi_{2}(e, \ell, g)=\frac{1}{2}\left[(\alpha-\beta) U_{m}(e) \sin (2 g+m \ell)\right. & \left.\quad+(\alpha+\beta) U_{3 m}(e) \sin (2 g+3 m \ell)\right], \\
\varphi_{3}(e, \ell, g)=\frac{1}{2}\left[(\alpha-\beta) U_{m}^{\prime}(e) \cos (2 g+m \ell)\right. & \left.+(\alpha+\beta) U_{3 m}^{\prime}(e) \cos (2 g+3 m \ell)\right] .
\end{aligned}
$$

Also, we note that $\delta$ in (15) plays the role of $\hat{\epsilon}$ in the lemma.

There are four cases to consider corresponding to the zero sets (18) of the unperturbed bifurcation function (14). We will consider the zero set $\mathcal{Z}_{1}^{+}$for illustrative purposes, as the computational procedure is identical in all four cases. The set $\mathcal{Z}_{1}^{+}$is just a line in $\mathbb{R}^{3}$, so here the curve $\Gamma$ in the lemma can be taken to be a parametrization of this line starting at an appropriate point. By a direct calculation, the function $\varphi_{2}-\varphi_{1}$ on $\mathcal{Z}_{1}^{+}$is given by

$$
\frac{1}{4}\left[(\alpha-\beta) U_{m}(e)+(\alpha+\beta) U_{3 m}(e)\right] \sin g .
$$

The zeros of this function along $\mathcal{Z}_{1}^{+}$are clearly simple provided the coefficient of $\sin g$ does not vanish at the value of the eccentricity determined by membership in $\mathcal{Z}_{1}^{+}$. The main result of this section is the following theorem.

Theorem V.1 Consider the system (10) and suppose that $(L, G, \ell, g)$ is on an unperturbed $(m: n)$ resonant periodic solution with $n=1$, that is, $L^{3}=m k^{2} / \Omega$. If $(e, \ell, g)$ is in $\mathcal{Z}_{1}^{+}$or $\mathcal{Z}_{1}^{-}$, respectively, $(e, \ell, g)$ is in $\mathcal{Z}_{2}^{+}$or $\mathcal{Z}_{2}^{-}$, where $e$ is the eccentricity of the corresponding Keplerian ellipse $\left(e^{2}=1-G^{2} / L^{2}\right)$ and if

$$
(\alpha-\beta) U_{m}(e)+(\alpha+\beta) U_{3 m}(e) \neq 0,
$$

respectively,

$$
(\alpha-\beta) U_{m}(e)-(\alpha+\beta) U_{3 m}(e) \neq 0,
$$

then the Keplerian ellipse continues to a periodic orbit under the perturbation.

It remains to show that the sets $\left\{\mathcal{Z}_{i}^{ \pm}\right\}, i=1,2$, are not empty. This fact will follow as soon as we show that both of the functions $U_{2 m}$ and $U_{2 m}^{\prime}$ have zeros on the interval $0<e<1$.

Recall that $U_{2 m}(e)=A_{2 m}(e)+B_{2 m}(e)$ and note that both $A_{2 m}$ and $B_{2 m}$ have removable singularities at $e=0$. Moreover, both have Taylor series at $e=0$ with leading terms given by

$$
2(2 m-1) \frac{m^{2 m-2}}{(2 m) !} e^{2(m-1)}
$$

In particular, if $m=1$, then $\lim _{e \rightarrow 0^{+}} U_{2}(e)=2$ and for $m>1$, the limit is zero, but $U_{2 m}(e)>0$ for sufficiently small eccentricity. Also, $\lim _{e \rightarrow 1^{-}} U_{2 m}=-J_{2 m}(2 m) / m^{2}$. By a standard property of the Bessel functions, $J_{\nu}(\nu)>0$; hence, $U_{2 m}(1)<0$. This proves $U_{2 m}$ has at least one zero on the interval $0<e<1$. Numerical calculations suggest that this zero is unique.

A simple argument can be given to prove the existence of a zero in the case $m=1$ for the function $U_{2 m}^{\prime}$. In this case, the series expansion of $U_{2}^{\prime}$ at $e=0$ is $-10 e+O\left(e^{2}\right)$. Thus, $U_{2}^{\prime}(e)<0$ for a small but positive eccentricity. The limit as $e \rightarrow 1^{-}$is the same as

$$
\lim _{e \rightarrow 1^{-}} \frac{2}{\sqrt{1-e^{2}}} J_{2}^{\prime}(2)
$$

By standard properties of the Bessel functions, $J_{2}(0)=0$ and $J_{2}>0$ on the interval $\left(0, j_{2,1}^{\prime}\right)$, where $j_{2,1}^{\prime}$ is the first zero of $J_{2}^{\prime}$. Hence, $J_{2}^{\prime}>0$ on $\left(0, j_{2,1}^{\prime}\right)$. But, we also have $2<j_{2,1}^{\prime}$. Thus, $J_{2}^{\prime}(2)>0$ and $U_{2}^{\prime}(e) \rightarrow \infty$ as $e \rightarrow 1^{-}$. This 
proves that $U_{2}^{\prime}(e)$ has at least one zero on the interval $0<e<1$; moreover, numerical computations suggest that this zero is unique.

For $m \geq 2$, we will outline a proof that shows the function $U_{2 m}^{\prime}$ has at least two zeros on the interval $0<e<1$. An asymptotic analysis shows that the function $U_{2 m}^{\prime}$ is positive near the end points of the interval. The proof is completed by showing that $U_{2 m}^{\prime}$ has a negative value within the interval. To this end, note that the function $U_{2 m}^{\prime}$ has the form

$$
U_{2 m}^{\prime}(e)=\gamma_{1}(m, e) J_{2 m}(2 m e)+\gamma_{2}(m, e) J_{2 m}^{\prime}(2 m e)
$$

where $\gamma_{1}$ and $\gamma_{2}$ are computed using (9). Moreover, by standard properties of the Bessel functions, both of the functions $e \mapsto J_{2 m}(2 m e)$ and $e \mapsto J_{2 m}^{\prime}(2 m e)$ are positive for $0<e<1$. Let us define $\widehat{e}:=\left(1-1 /\left(4 m^{2}\right)\right)^{1 / 2}$, and observe that $0<\widehat{e}<1$. An simple computation shows that

$$
\gamma_{1}(m, \widehat{e})<0, \quad \gamma_{2}(m, \widehat{e})<0 .
$$

Hence, we have $U_{2 m}^{\prime}(\widehat{e})<0$, as desired. Numerical computations suggest that the function $U_{2 m}^{\prime}$ has exactly two zeros on the interval $0<e<1$.

It follows from these results that the resonant interaction of the incident multi-frequency gravitational wave of definite helicity with a Keplerian binary can result in orbits that are periodic with period $2 \pi / \Omega$, where $\Omega=m \omega$, $m=1,2,3, \ldots$. It is conceivable that other periodic orbits may exist; however, our method can identify only those periodic orbits that are continuations of resonant Keplerian orbits of the unperturbed system.

\section{ROTATING FRAME}

In a manner similar to that used by Hill in his treatment of the lunar theory 9 , we can view the dynamical system described by (6) in Cartesian coordinates rotating at half the principal frequency of the incident gravitational wave $\left(\Omega_{\star}=2 \Omega\right)$. These coordinates - that we again represent by $(x, y)$-rotate with frequency $\frac{1}{2} \Omega_{\star}=\Omega$ with respect to inertial coordinates; therefore, the equations of motion in these coordinates are given by

$$
\begin{gathered}
\frac{d^{2} x}{d t^{2}}-2 \Omega \frac{d y}{d t}-\Omega^{2} x+\frac{k x}{r^{3}}+2 \epsilon \Omega^{2}[(1+\delta \alpha \cos \Omega t) x \\
+(\delta \beta \sin \Omega t) y]=0 \\
\frac{d^{2} y}{d t^{2}}+2 \Omega \frac{d x}{d t}-\Omega^{2} y+\frac{k y}{r^{3}}+2 \epsilon \Omega^{2}[(\delta \beta \sin \Omega t) x \\
-(1+\delta \alpha \cos \Omega t) y]=0 .
\end{gathered}
$$

Let $X:=\dot{x}-\Omega y$ and $Y:=\dot{y}+\Omega x$ be the canonical momenta conjugate to $x$ and $y$, respectively. Then (19) is equivalent to a Hamiltonian system with Hamiltonian

$$
\begin{aligned}
\mathcal{H}_{R}= & \frac{1}{2}\left(X^{2}+Y^{2}\right)+\Omega(y X-x Y)-\frac{k}{r} \\
& +\epsilon \Omega^{2}\left[(1+\delta \alpha \cos \Omega t)\left(x^{2}-y^{2}\right)+2(\delta \beta \sin \Omega t) x y\right] .
\end{aligned}
$$

By identifying the momenta in polar rotating coordinates as $p_{r}=(x X+y Y) / r$ and $p_{\theta}=x Y-y X$, (20) in polar coordinates is given by

$$
\begin{aligned}
\mathcal{H}_{R}=\frac{1}{2}\left(p_{r}^{2}+\frac{p_{\theta}^{2}}{r^{2}}\right) & -\frac{k}{r}-\Omega p_{\theta}+\epsilon \Omega^{2} r^{2}[\cos 2 \theta \\
& +\delta(\alpha \cos 2 \theta \cos \Omega t+\beta \sin 2 \theta \sin \Omega t)]
\end{aligned}
$$

which in the corresponding Delaunay elements becomes

$$
\begin{aligned}
\mathcal{H}_{R}=-\frac{k^{2}}{2 L^{2}}-\Omega G+\epsilon \Omega^{2}\{\mathcal{C}(L, G, \ell, g) & +\delta[\alpha \mathcal{C}(L, G, \ell, g) \cos \Omega t \\
& +\beta \mathcal{S}(L, G, \ell, g) \sin \Omega t]\}
\end{aligned}
$$

The Hamiltonian system given by (22) has periodic orbits with period $2 \pi / \Omega$, where $\Omega=m \omega, m=1,2, \cdots ;$ this assertion can be demonstrated using results that have already been obtained in this paper. In fact, using the resonance 
assumption, periodic orbits exist in the inertial frame with period $2 \pi / \Omega$ as shown in Section $\bigvee$. An orbit in the inertial frame, $t \mapsto\left(x_{I}(t), y_{I}(t)\right)$, is represented in the rotating frame by

$$
\begin{aligned}
& x_{R}(t)=x_{I}(t) \cos \Omega t+y_{I}(t) \sin \Omega t, \\
& y_{R}(t)=-x_{I}(t) \sin \Omega t+y_{I}(t) \cos \Omega t .
\end{aligned}
$$

It follows from these relations that if $x_{I}(t)$ and $y_{I}(t)$ are periodic with period $2 \pi / \Omega$, then so are $x_{R}(t)$ and $y_{R}(t)$. Therefore, the orbits that are periodic in the inertial frame with period $2 \pi / \Omega$ are observed to be periodic with period $2 \pi / \Omega$ in the rotating frame. It is also possible to arrive at this conclusion by direct application of the methods developed in the previous sections.

To understand the physical structure of Hamiltonian (22), imagine right circularly polarized gfavitational radiation of frequency $2 \Omega$ that is normally incident on the orbital plane. It follows from our previous work 1 , as well as equation (19), that in a reference frame rotating with frequency $\Omega$ the wave stands still. That is, observers at rest in the rotating frame do not perceive the variability associated with a wave so that in the absence of secondaries $(\delta=0)$ the dynamical system (19) is autonomous. That an observer - by merely rotating about the propagation axis of the circularly polarized wave - could make the wave stand completely still would be a remarkable physical effect and deserves further discussion.

It is a fundamental consequence of Lorentz invariance that all basic radiation fields travel with speed $c$ with respect to all inertial observers. This may be illustrated by an example: Let an inertial observer move with speed $v_{0}$ along the propagation axis of a monochromatic plane gravitational wave of frequency $\Omega_{\star}$. The frequency and the wave vector of the radiation as perceived by the moving observer are smaller than those measured by static inertial observers by a common Doppler factor of

$$
\left(\frac{c-v_{0}}{c+v_{0}}\right)^{\frac{1}{2}} .
$$

Mathematically, as $v_{0} \rightarrow c$ this ratio goes to zero and hence the frequency and wave vector of the radiation vanish so that the wave might appear to stand still. This limit is not physically allowed, however. No observer can move at the speed of light, although - theoretically - one can get arbitrarily close. Therefore, the wave can never stand still for an inertial observer. It has been shown 10 that according to the standard Einstein theory this is not the case for accelerated observers, i.e. an accelerated observer can indeed stand still with respect to a gravitational wave. The autonomous nature of the system (19) for $\delta=0$ provides an interesting illustration of this fact. That is, consider an observer at the center of a system of coordinates rotating with frequency $\Omega$. The observer does not move, but the fact that it refers its observations to the axes that rotate with frequency $\Omega$ with respect to the inertial axes makes it a noninertial observer. Radiation of frequency $\Omega_{\star}$ is incident in the inertial frame along the axis of rotation. According to the noninertial observer, the frequency of the gravitational wave is $\Omega_{\star}^{\prime}=\Omega_{\star} \mp 2 \Omega$, where the upper sign refers to right circularly polarized (RCP) gravitational radiation and the lower sign refers to left circularly polarized (LCP) gravitational radiation. The first (second) case has helicity $+2(-2)$, so that $\Omega_{\star}^{\prime}=\Omega_{\star}-\mathbf{h} \cdot \boldsymbol{\Omega}$, where $\mathbf{h}$ is the helicity of the gravitational radiation field; this is an example of the general phenomenon of helicity-rotation coupling. Now if $\Omega=\frac{1}{2} \Omega_{\star}$ for RCP gravitational waves or $\Omega=-\frac{1}{2} \Omega_{\star}$ for LCP gravitational waves, we find that $\Omega_{\star}^{\prime}$ vanishes according to the noninertial observer (as well as any other observer at rest in the rotating frame anywhere along the $z$-axis) and the radiation field stands still. It has been shown in Ref. 1 that for a monochromatic gravitational wave with definite helicity and sufficiently small amplitude this observation concerning a rotating observer leads to the conclusion that a Keplerian system in the presence of this radiation can never ionize. In principle, this absence of ionization could be considered an observable consequence of the physical possibility that a gravitational wave could stand completely still.

Let us now consider the possibility of ionization of the binary system as a function of the parameter $\delta$. The transformation to the rotating frame leaves the orbital radius unchanged; therefore, the ionization problem can be discussed equally well in the rotating frame. In fact, the problem becomes simpler since the principal component of the incident radiation field loses its time - dependence as in (22). Thus if $\delta=0$, the KAM theorem implies that for sufficiently small $\epsilon$ the perturbed trajectory is bounded since it is trapped between two-dimensional invariant tori in the three-dimensional energy surfaces. When $\delta \neq 0$, however, the two secondary components in the inertial frame both reduce to a perturbation of frequency $\Omega$ in the rotating frame; that is, the three Fourier components of the radiation field in the inertial frame are RCP waves with frequencies $\Omega_{\star}=\Omega, 2 \Omega$ and $3 \Omega$, while the tidal matrix in the rotating frame has frequencies given by $\Omega_{\star}^{\prime}=\Omega_{\star}-2 \Omega$, i.e. $-\Omega, 0$ and $\Omega$ for the three compenents, respectively. The perturbation of frequency $\Omega$ in the rotating frame is expected to lead to Arnold diffusion 11.12 and hence ionization of the system. However, it has not been possible thus far to prove ionization for the system (22); therefore, we resort to numerical work in the following section. 


\section{NUMERICAL EXPERIMENTS}

In this section we illustrate - by means of numerical experiments - the conjecture that gravitational ionization and Arnold diffusion are closely related. The interpretation of the numerical results is simplified if we take the viewpoint of inertial observers and consider the perturbed motion given by Hamiltonian (6).

We have performed several numerical experiments to test the diffusion and ionization properties of the dynamical system that is represented by the Hamiltonian (6). The physical meaning of these numerical experiments is essentially the same as in our previous paper (cf. Figure 2 in Ref. 11): Let us choose two scales for the measurement of time and length that are arbitrary except that they are connected here by our choice of $k=1$. In these otherwise unspecified units, we have chosen an unperturbed ellipse of semimajor axis $a=4 / 3$ and eccentricity $e=1 / 2$ such that $g=-\pi / 2$ and the Keplerian frequency is $\omega=3 \sqrt{3} / 8$. The ellipse is perturbed by the presence of periodic gravitational radiation and the orbital radius of the osculating ellipse is then plotted versus time in these units in Figures 1-4. These figures represent the results of our numerical experiments in which we have set the parameters of the external perturbation as follows:

$$
\epsilon=.001, \quad \alpha=2.5, \quad \beta=2,
$$

and have changed $\delta$ in the range $0 \leq \delta \leq 1$ and $\Omega$ in the range $1 \leq \Omega \leq 2$. In each run, we set the initial conditions to be

$$
\left(p_{r}, p_{\theta}, r, \theta\right)=(.5,1,1,0),
$$

which correspond to the unperturbed ellipse described above. After integration over each time interval corresponding to one cycle of the perturbation, i.e. $2 \pi / \Omega$, the corresponding elapsed time $t$ and orbital radius are plotted. Clearly, for each value of $t$ (abscissa) there is only one value of orbital radius $r$ (ordinate); however, this is not discernible in some of the figures due to the way in which the plots have been prepared. The KAM theorem is illustrated in Figure 1 , where the value of $r$ appears to oscillate between $\simeq a(1-e)=2 / 3$ and $\simeq a(1+e)=2$, thus indicating the complete absence of ionization. The possibility of ionization of the system is illustrated in Figures 2 and 3 for $\delta>0$, where the initial ellipse is near resonance in the top panel, on resonance in the middle panel and off resonance in the bottom panel. More precisely, the middle panel in either Figure 2 or Figure 3 corresponds to an exact third-order resonance since $\Omega / \omega=3$, while the top panel illustrates the response of the ellipse to the external perturbation near resonance $\Omega / \omega \approx 3.08$; the bottom panel is off resonance with $\Omega / \omega \approx 1.54$. Additional calculations extending the integration time for the system depicted in the top panel of Figure 3 have been performed. These results suggest that a bursting behavior occurs in which the near resonance condition shown at the right end of this panel is followed by chaotic motion similar to that shown in the middle of this panel that in turn is followed by a period of near resonance. This recurrence of chaotic and near resonance behavior appears to continue for the extended interval of time studied as illustrated in Figure 4. Thus, this behavior is consistent with a type of chaotic behavior, called intermittent chaos, that has been studied for dissipative systems 12 . If the behavior suggested by these simulations is indeed present in the Hamiltonian system (6), then our result would be an example of Hamiltonian intermittency.

Imagine, for the sake of concreteness, a binary system consisting of an artificial satellite in an eccentric orbit about the Earth. Let the scales of length and time be $R_{0}$ and $T_{0}$, respectively; then, $R_{0}^{3}=k T_{0}^{2}$. Thus, if we take $R_{0}=10^{9} \mathrm{~cm}$ for the problem under consideration, it turns out that $T_{0} \approx 1.6 \times 10^{3}$ sec. The gravitational wave in our numerical experiments would then have a frequency of the order of $\Omega \approx 10^{-3} \mathrm{rad} \mathrm{sec}^{-1}$, corresponding approximately to $1.5 \times 10^{-4} \mathrm{~Hz}$ as well as to a wavelength of $2 \times 10^{14} \mathrm{~cm}$, and an amplitude of the order of $\epsilon=10^{-3}$. Gravitational waves have not yet been directly observed; however, in a realistic situation the amplitude of the wave would be expected to be of the order of $10^{-20}$.

Our numerical experiments are consistent with the expected behavior for a $2 \frac{1}{2}$-degree of freedom Hamiltonian system. In fact, by introducing a fictitious action variable (cf. $\S 6$ in Ref. 1), our system is equivalent to an autonomous Hamiltonian system with three degrees of freedom. Each orbit of this new system is constrained to an energy manifold. However, whereas the two dimensional KAM tori (if they exist for our choice of parameter values) separate each three dimensional energy manifold for the corresponding two-degree of freedom Hamiltonian system that we obtain with $\delta=0$, the three dimensional KAM tori, that may exist for the three-degree of freedom Hamiltonian system that we obtain with $\delta \neq 0$, do not separate space within the five dimensional energy manifolds. Of course, for sufficiently small choices of $\delta$, there are orbits of the three-degree of freedom Hamiltonian system that remain bounded for all time; for example, the periodic orbits of Section $\mathrm{Q}$ and the orbits confined to KAM tori. While the totality of bounded orbits in an energy manifold may be a set of positive measure, we expect that every open set of the five dimensional energy manifold contains an initial condition for a trajectory that will diffuse throughout the energy manifold. In fact, we expect this behavior for all $\delta>0$. On the other hand, as $\delta$ decreases toward zero, the time required to leave the vicinity of a KAM torus is expected to grow at an exponential rate. Figures 1-4 illustrate dynamical behavior that is characteristic of Arnold diffusion 12 . 


\section{APPENDIX}

The purpose of this appendix is to point out that the main results of this paper still hold for a more general incident gravitational wave than that considered in (4).

In the general case of incident gravitational radiation on a binary system, the motion of the system away from the initial orbital plane (i.e. along the $z$-direction) also needs to be taken into account. It is necessary to mention here that initial conditions are generally ignored in our theoretical approach, which relies on the properties of the perturbed system once transients have died away and a "steady state" situation has been established. In this paper, we have limited our considerations to normally incident waves; in fact, the transversality of gravitational radiation makes it possible to set $z=0$ for normally incident waves. We wish to note here that essentially the same results can be obtained for a more general incident radiation field.

Consider, for instance, the superposition of a left circularly polarized (LCP) wave of frequency $\Omega$ traveling along the $z$-axis with a linearly polarized wave of frequency $\Omega$ traveling along the $x$-axis. Specifically, let $\chi_{i j}$ be of the form

$$
\chi(t, \mathbf{0})=\left(\begin{array}{ccc}
\cos \Omega t & -\sin \Omega t & 0 \\
-\sin \Omega t & 3 \cos \Omega t & 0 \\
0 & 0 & -4 \cos \Omega t
\end{array}\right)
$$

up to a constant factor. It follows from (2) that the corresponding $\mathcal{K}$ is of the form

$$
\mathcal{K}=\frac{1}{2} \Omega^{2} \chi(t, \mathbf{0})
$$

so that the equation of motion (1) along the $z$-direction is given by

$$
\frac{d^{2} z}{d t^{2}}+\frac{k z}{r^{3}}-2 \epsilon \Omega^{2} z \cos \Omega t=0 .
$$

This equation is satisfied by $z=0$, which is consistent with the fact that the orbit is always in the $(x, y)$-plane. Therefore, to the incident radiation field in (4) one could add radiation fields of the type given by (23). Moreover, upon transformation to the rotating frame

$$
\mathcal{K}^{\prime}=R^{-1} \mathcal{K} R=\frac{1}{2} \Omega^{2}\left(\begin{array}{ccc}
\cos \Omega t & \sin \Omega t & 0 \\
\sin \Omega t & 3 \cos \Omega t & 0 \\
0 & 0 & -4 \cos \Omega t
\end{array}\right)
$$

where

$$
R=\left(\begin{array}{ccc}
\cos \Omega t & -\sin \Omega t & 0 \\
\sin \Omega t & \cos \Omega t & 0 \\
0 & 0 & 1
\end{array}\right)
$$

is the rotation matrix used in Section VII. It is remarkable that the tidal matrix $\mathcal{K}^{\prime}$ in the rotating frame has the same frequency $\Omega$ as in the inertial frame; in fact, $\mathcal{K}^{\prime}$ can be obtained from $\mathcal{K}$ simply by letting $\Omega \rightarrow-\Omega$.

${ }^{1}$ C. Chicone, B. Mashhoon and D. G. Retzloff, Gravitational ionization: periodic orbits of binary systems perturbed by gravitational radiation, Annales de l'Institut Henri Poincaré, Physique Théorique, 64, 87-125 (1996).

${ }^{2}$ C. Chicone, A geometric approach to regular perturbation theory with an application to hydrodynamics, Trans. AMS, 347, 4559-4598 (1995).

${ }^{3}$ B. Mashhoon, Tidal radiation, Astrophys. J., 216, 591-609 (1977).

${ }^{4}$ B. Mashhoon, On tidal resonance, Astrophys. J., 223, 285-298 (1978).

${ }^{5}$ R. A. Hulse and J. H. Taylor, Discovery of a pulsar in a binary system, Astrophys. J., 195, L51-53 (1975).

${ }^{6}$ J. H. Taylor, A. Wolszczan, T. Damour and J. M. Weisberg, Experimental constraints on strong-field relativistic gravity, Nature, 355, 132-136 (1992).

7 J. Kovalevsky, Introduction to Celestial Mechanics, Astrophysics and Space Science Library, Vol. 7, Springer-Verlag, 1967. 
${ }^{8}$ H. Poincaré, Les Méthodes Nouvelles de la Mécanique Céleste, Vols. 1-3, Gauthier-Villars, Paris, $1892-99$.

${ }^{9}$ S. Sternberg, Celestial Mechanics, Vols. 1-2, W. A. Benjamin, Inc., New York, 1969.

${ }^{10}$ B. Mashhoon, Accelerated observers and conformal invariance, in: Quantum Gravity and Beyond: Essays in Honor of Louis Witten, edited by F. Mansouri and J. J. Scanio, World Scientific, Singapore, 1993, pp. 257-271.

${ }^{11}$ V. I. Arnold, Small denominators and problems of stability of motion in classical and celestial mechanics, Russian Math. Surveys, 18, 85-192 (1963).

${ }^{12}$ A. J. Lichtenberg and M. A. Lieberman, Regular and Chaotic Dynamics, Springer-Verlag, New York, 1992.

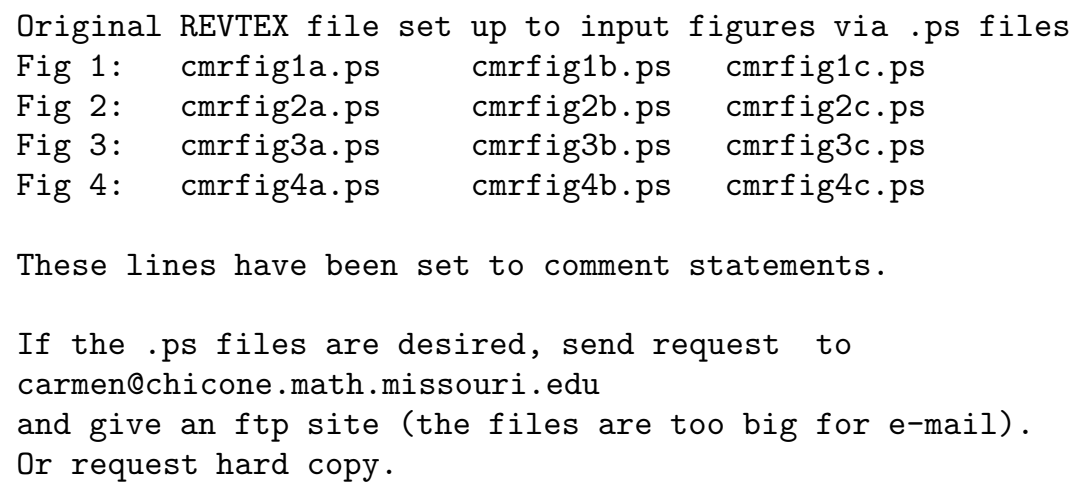

FIG. 1. Orbital radius versus time plots for the dynamical system given by Hamiltonian (6) with initial conditions $\left(p_{r}, p_{\theta}, r, \theta\right)$ equal to $(.5,1,1,0)$ and parameter values $\epsilon=.001, \delta=0, \alpha=2.5, \beta=2, k=1$, and, from top to bottom, $\Omega=2, \Omega=9 \sqrt{3} / 8, \Omega=1$.

FIG. 2. Orbital radius versus time plots for the dynamical system given by the Hamiltonian (6) with initial conditions $\left(p_{r}, p_{\theta}, r, \theta\right)$ equal to $(.5,1,1,0)$ and parameter values $\epsilon=.001, \delta=0.5, \alpha=2.5, \beta=2, k=1$, and, from top to bottom, $\Omega=2$, $\Omega=9 \sqrt{3} / 8, \Omega=1$.

FIG. 3. Orbital radius versus time plots for the dynamical system given by the Hamiltonian (6) with initial conditions $\left(p_{r}, p_{\theta}, r, \theta\right)$ equal to $(.5,1,1,0)$ and parameter values $\epsilon=.001, \delta=1, \alpha=2.5, \beta=2, k=1$, and, from top to bottom, $\Omega=2$, $\Omega=9 \sqrt{3} / 8, \Omega=1$.

FIG. 4. Orbital radius versus time plots for the dynamical system given by the Hamiltonian (6) with initial conditions $\left(p_{r}, p_{\theta}, r, \theta\right)$ equal to $(.5,1,1,0)$ and parameter values $\epsilon=.001, \delta=0.5, \alpha=2.5, \beta=2, k=1, \Omega=2$. This is an extended form of the plot presented in the top panel of Figure 3 . 